\title{
The influence of central review on outcome in malignant gliomas of the spinal cord: the CCG-945 experience
}

\author{
Eric Bouffet, MD,, Jeffrey C. Allen, MD, ${ }^{2}$ James M. Boyett, PhD, ${ }^{3}$ Allen Yates, MD, ${ }^{4}$ \\ Floyd Gilles, MD, ${ }^{5}$ Peter C. Burger, MD, ${ }^{6}$ Richard L. Davis, MD, ${ }^{7}$ Laurence E. Becker, MD, ${ }^{8}$ \\ lan F. Pollack, MD, ${ }^{9}$ and Jonathan L. Finlay, $\mathrm{MBChB}^{10}$
}

\begin{abstract}
${ }^{1}$ The Hospital for Sick Children, University of Toronto, Ontario, Canada; ${ }^{2}$ New York University Langone Medical Center, New York, New York; ${ }^{3}$ Biostatistics, Saint Jude Children's Research Hospital, Memphis, Tennessee; ${ }^{4}$ Department of Pathology, The Ohio State University, Columbus, Ohio; ${ }^{5}$ Department of Pathology, Children's Hospital Los Angeles, California; ${ }^{6}$ Department of Pathology, Johns Hopkins University School of Medicine, Baltimore, Maryland; 'Department of Pathology, University of California, San Francisco, California; ${ }^{8}$ Department of Pathology, The Hospital for Sick Children, University of Toronto, Ontario, Canada; 'Department of Neurosurgery, Children's Hospital of Pittsburgh, Pennsylvania; and ${ }^{10}$ Pediatric Nationwide Children's Hospital, Columbus, Ohio
\end{abstract}

OBJECTIVE The impact of central pathology review on outcome has been described in pediatric patients with highgrade glioma (HGG). The objective of this report was to analyze the impact of the central pathology review on outcome in the subgroup of patients with institutional diagnosis of HGG of the spinal cord enrolled in the Children's Cancer Group 945 cooperative study.

METHODS Five neuropathologists centrally reviewed the pathology of the 18 patients with HGG of the spinal cord who were enrolled in the study. These reviews were independent, and reviewers were blinded to clinical history and outcomes. A consensus diagnosis was established for each patient, based on the outcome of the review.

RESULTS Of 18 patients, only 10 were confirmed to have HGG on central review. At a median follow-up of 12 years, event-free and overall survival for all 18 patients was $43.2 \% \pm 13.3 \%$ and $50 \% \pm 13.4 \%$, respectively. After central review, 10-year event-free and overall survival for confirmed HGGs and discordant diagnoses was $30 \% \pm 12.5 \%$ versus $58.3 \% \pm 18.8 \%(p=0.108)$ and $30 \% \pm 12.5 \%$ versus $75 \% \pm 14.2 \%(p=0.0757)$, respectively.

CONCLUSIONS The level of discordant diagnoses in children and adolescents with institutional diagnosis of HGG of the spinal cord was $44 \%$ in this experience. However, there was no significant difference in outcome between patients with confirmed and discordant diagnosis. This group of tumor deserves a specific attention in future trials.

http://thejns.org/doi/abs/10.3171/2015.10.PEDS1581

KEY WORDS malignant glioma; spinal cord; pathology; central review; spine; oncology

$\mathrm{T}$ HE difficulty of classifying pediatric high-grade gliomas (HGGs) has been illustrated by the outcome of the central pathology review conducted in the Children's Cancer Group 945 study (CCG-945), the largest randomized study reported to date of pediatric malignant gliomas. ${ }^{6}$ The results of this review have been reported and have demonstrated frequent discordance between the local institutional diagnosis and the outcome of central pathology review..$^{15}$ The initial central pathology review was conducted by one expert prior to the 1993 WHO revision, and 24 tumors were considered to be overtly discordant with a diagnosis of HGG. Using the revised WHO criteria, ${ }^{11}$ a panel of 5 neuropathologists identified 51 of 172 tumors as being discordant. ${ }^{15}$ There was also discordance among expert reviewers, ${ }^{8}$ and this suggests that, despite availability of established diagnostic criteria, ${ }^{11}$ applying WHO guidelines in a large prospective cooperative study can be challenging. Issues concerning central pathology review for some specific subgroups of the CCG-945 study have been previously reported. ${ }^{7,9}$

ABBREVIATIONS AA = anaplastic astrocytoma; CCG = Children's Cancer Group; EFS = event-free survival; GBM = glioblastoma multiforme; HGG = high-grade glioma; OS = overall survival.

SUBMITTED February 4, 2015. ACCEPTED October 5, 2015.

INCLUDE WHEN CITING Published online December 18, 2015; DOI: 10.3171/2015.10.PEDS1581. 
Intrinsic spinal cord tumors comprise only 3\%-5\% of pediatric central nervous system neoplasms ${ }^{5}$ and include intra- and extramedullary lesions of various histologies that largely mirror the normal composition of the spine and associated coverings. Among intramedullary tumors, malignant astrocytomas are reported to represent $10 \%$ of spinal tumors in most institutional series. Higher proportions $(30 \%-50 \%)$ reported in uni- or multiinstitutional studies $^{1,2,19}$ may reflect referral biases and difference in histopathological grading criteria. The aim of the present report is to describe the unique outcome of central pathology-reviewed patients with malignant glioma of the spinal cord who were enrolled in the CCG-945 protocol.

\section{Methods}

As in the predecessor study CCG- $943,{ }^{22}$ patients with primary spinal cord lesions were initially not eligible for enrollment in CCG-945 until an amendment was issued, allowing children with primary spinal cord HGG to be enrolled. From December 1986 to May 1991, 18 patients with high-grade astrocytoma arising within the spinal cord were registered in CCG-945. The protocol recommended resection of as much tumor as safely feasible without jeopardizing the patient. In the main study, postoperative treatment consisted of a combination of radiotherapy and chemotherapy with either adjuvant prednisone, lomustine, and vincristine (the control regimen) or the 8-drugs-in-1day regimen (an experimental regimen that included highdose methylprednisolone and 7 agents with confirmed or suspected activity against pediatric brain tumors: lomustine, vincristine, hydroxyurea, procarbazine, cisplatin, cytosine arabinoside, and dacarbazine), administered for 2 cycles before irradiation and continued after irradiation. However, children with primary spinal cord tumors were nonrandomly assigned to the experimental " 8 -in-1" regimen, along with craniospinal irradiation and boost to the primary tumor site. Radiotherapy was to be given concomitantly with the second cycle. Children older than 3 years with focal lesions were to receive craniospinal axis irradiation up to a dose of 3600 cGy with an 1800-cGy boost to the primary tumor, whereas children with evidence of leptomeningeal dissemination were to receive 3600 cGy to the craniospinal axis and a $1440-\mathrm{cGy}$ boost to the entire spinal cord. For children between 24 and 36 months of age, treating physicians were offered 2 therapeutic options: either fractionated radiotherapy at a total dose of $2340 \mathrm{cGy}$ to the craniospinal axis with a 2160-cGy boost to the primary site after 10 cycles of chemotherapy; or local treatment at a dose of $4500 \mathrm{cGy}$ to the primary tumor site after 2 cycles of 8 -in-1. Children younger than 24 months at the time of diagnosis could be treated with 10 cycles of 8-in-1 chemotherapy alone to delay or possibly avoid radiotherapy. Details regarding the characteristics and management of each patient were provided in the initial publication. However, the results for this subgroup were initially reported prior to the conduct of the centralized consensus pathology review. ${ }^{1}$

\section{Central Pathology Review}

Central pathology review was requested for all tumors from patients entered into the study. Specimens were reviewed initially by one study neuropathologist. However, 1 year after completion of the CCG-945 study, the WHO published a revised classification of pediatric brain tumors. ${ }^{11}$ The introduction of new diagnostic criteria raised questions regarding patients treated in CCG-945 and prompted a more comprehensive pathology review based on the revised 1993 WHO classification for pediatric brain tumors. For the purpose of this second review, each case was masked and independently reviewed by 5 experienced neuropathologists who were asked to classify the tumors based on the revised criteria. The details of the methodology used for this central review were previously reported. ${ }^{8}$ Tumors were classified as glioblastoma multiforme (GBM), anaplastic astrocytoma (AA), other eligible HGGs, and discordant (not HGG). Specimens were labeled "concordant" when at least 3 of 5 pathologists confirmed the diagnosis of HGG. When a different diagnosis was proposed, a consensus diagnosis was provided when 3 or more pathologists agreed on the revised histology.

\section{Biological Studies}

Because status of TP53 mutations, p53 expression, the presence of alterations to chromosome arms $1 p$ and $19 q$, and tumor proliferation index each have been associated with outcome in children with HGGs within the entire CCG-945 cohort, ${ }^{16-18}$ information specific to the subgroup of patients with spinal cord astrocytoma was retrieved from the main biological database.

\section{Statistical Analysis}

Overall survival (OS) and event-free survival (EFS) were analyzed. OS was calculated as the time from date of diagnosis to date of death. Patients still alive were censored at date last seen. EFS was calculated as the time from date of diagnosis to the date of first event. An event was defined as recurrence, tumor progression/dissemination, or death. In those cases in which death followed recurrence, the event was the recurrence. Distributions of EFS and OS were estimated using the Kaplan-Meier method. Standard errors were derived from Greenwood's formula. Differences in survival among patient groups were evaluated using the log-rank test.

\section{Results}

\section{Histopathology Review}

Eighteen children were enrolled (11 males and 7 females, median age at diagnosis 7.7 years, range 12 months-17.7 years). Institutional diagnoses were AA (12 patients), GBM (4 patients), and anaplastic mixed glioma (2 patients). After central review, there was a consensus on the diagnosis of HGG for 10 patients, and 8 diagnoses were discordant. Six patients were diagnosed with AA and 4 with GBM. Eight patients were not eligible for the following reasons: low-grade glioma $(n=6$, including 2 juvenile pilocytic astrocytomas, 3 fibrillary astrocytomas, and 1 low-grade oligodendroglioma), ependymoma $(\mathrm{n}=1)$, and insufficient material $(\mathrm{n}=1)$. However, there was a striking variance among pathologists in the proportion of the 18 patients who had a discordant final diagnosis (Table 1). The insti- 


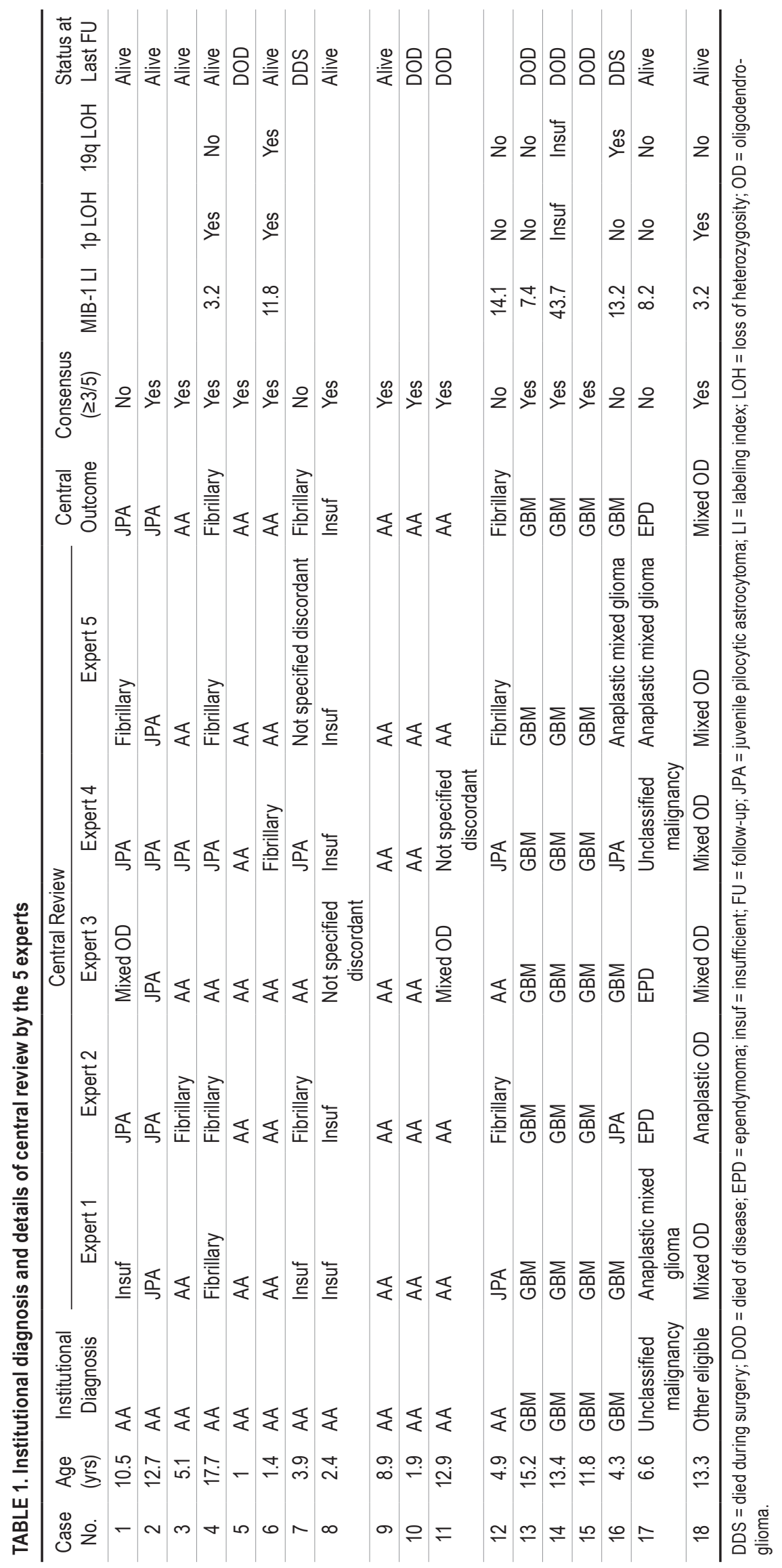


tutional diagnosis of HGG was confirmed 12 times (67\%) by 1 expert, and only $10,9,8$, and 7 times by the other experts. There was full agreement among reviewers in 7 cases (3 GBM, 3 AA, and 1 low-grade glioma), among 4 of 5 reviewers in 3 cases (1 insufficient material, 1 AA, 1 mixed glial tumor not eligible), and among 3 of 5 reviewers in 3 cases (2 AA and 1 fibrillary glioma). Overall, 8 of 18 registered patients were deemed not eligible $(n=7)$ or not amenable to consensus because of insufficient material (n $=1$ ). In addition, 1 patient with GBM on consensus review was considered progressive after 1 cycle of chemotherapy and underwent gross-total resection of his tumor. The institutional diagnosis at the second resection was that of anaplastic ganglioglioma.

\section{Biological Studies}

Specimens from 14 of the 18 patients were available for analysis. However, specimens were deemed usable for only 8 patients, including 4 with confirmed HGG. Seven specimens could be assessed for p53 expression, and 6 contained sufficient tissue for analysis of TP53 mutations. Seven tumors showed little or no expression of p53 and only one, considered low-grade glioma on central pathology review, showed dense p53 immunoreactivity. None of the 6 tumors (including 2 GBMs and 1 AA) examined exhibited a mutation of TP53. Proliferation index, as assessed by MIB-1 antibody labeling of the nuclear Ki 67 antigen, ranged between $3.2 \%$ and $43.2 \%$ in the 8 specimens studied. Four patients with confirmed HGG had indices of $7.4 \%, 11.8 \%, 13.2 \%$, and $43.2 \%$, whereas 4 patients with discordant nonmalignant histologies had indices of $3.2 \%, 8.2 \%, 8.8 \%$, and $14.1 \%$. Six tumors were assessable for both $1 \mathrm{p}$ and $19 \mathrm{q}$, of which 3 had deletions: one low-grade glioma had 1p deletion, one GBM harbored $19 q$ deletion, and one AA had both $1 p$ and $19 q$ deletions. All patients with $1 p$ and/or $19 q$ deletions eventually progressed and died of tumor progression.

\section{Revised Outcome}

At the last follow-up on June 2010, 9 patients were alive, including 2 patients who exhibited further tumor progression 78 and 97 months after diagnosis. Nine patients died, 7 of tumor progression and 2 while undergoing surgery for severe kyphosis. None of the confirmed GBM patients survived. However, one of these patients (with pathology reviewed at second surgery of anaplastic ganglioglioma) died during kyphosis surgery 33 months after diagnosis. Three of the 6 patients with confirmed AA are alive. In the group of patients with discordant histology, 6 patients are alive and 2 patients died, including 1 during kyphosis surgery. At a median follow-up of 12 years, EFS and OS for all 18 patients is $43.2 \% \pm 13.3 \%$ and $50 \% \pm 13.4 \%$, respectively (Fig. 1). After central review, 10-year EFS and OS for confirmed HGG and discordant diagnoses was $30 \% \pm 12.5 \%$ versus $58.3 \% \pm 18.8 \%(\mathrm{p}=0.108)$ and $30 \%$ $\pm 12.5 \%$ versus $75 \% \pm 14.2 \%(\mathrm{p}=0.0757)$, respectively (Figs. 2 and 3). Overall survival and EFS between confirmed HGG and discordant diagnoses were not significantly different for each individual pathologist (data not shown). Age younger than 5 years at diagnosis was the

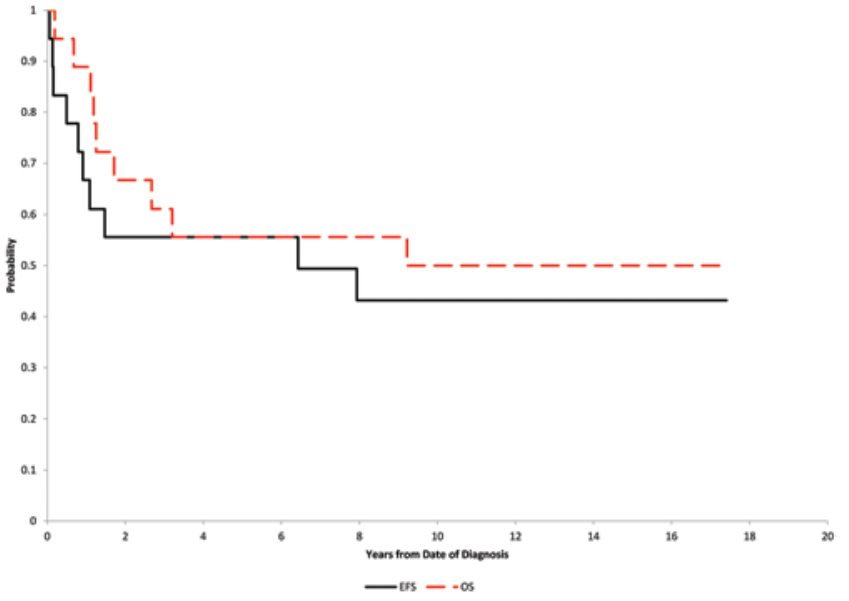

FIG. 1. Event-free survival and OS for 18 eligible patients. Figure is available in color online only.

only factor associated with poorer OS and EFS ( $p=0.05$ and $\mathrm{p}=0.01$, respectively).

\section{Discussion}

Reports on malignant spinal gliomas are rare, reflecting the low incidence of this entity, and CCG-945 remains to date the largest prospective study that has included pediatric patients with HGG of the spinal cord. Whether the population enrolled in this protocol truly reflects the characteristics of pediatric patients with HGG of the spinal cord is unknown. However, CCG-945 was the only protocol open during that period for pediatric patients with HGG in North America and there was therefore no competing study. These tumors are aggressive, with a propensity to disseminate, and survival is poor. In an institutional review of 19 pediatric and adult patients with malignant astrocytoma of the spinal cord, only 4 patients were alive at the time of publication, and the median survival time was 6 months. ${ }^{4}$ In a retrospective series of 73 pediatric patients with spinal cord astrocytoma, Bouffet et al. reported

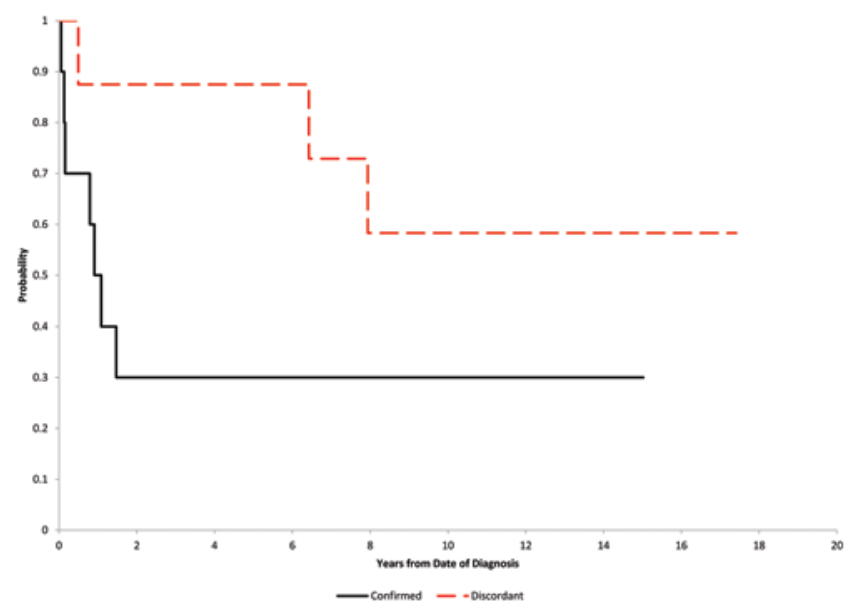

FIG. 2. Distributions of EFS by confirmed diagnosis and discordant diagnosis. Figure is available in color online only. 


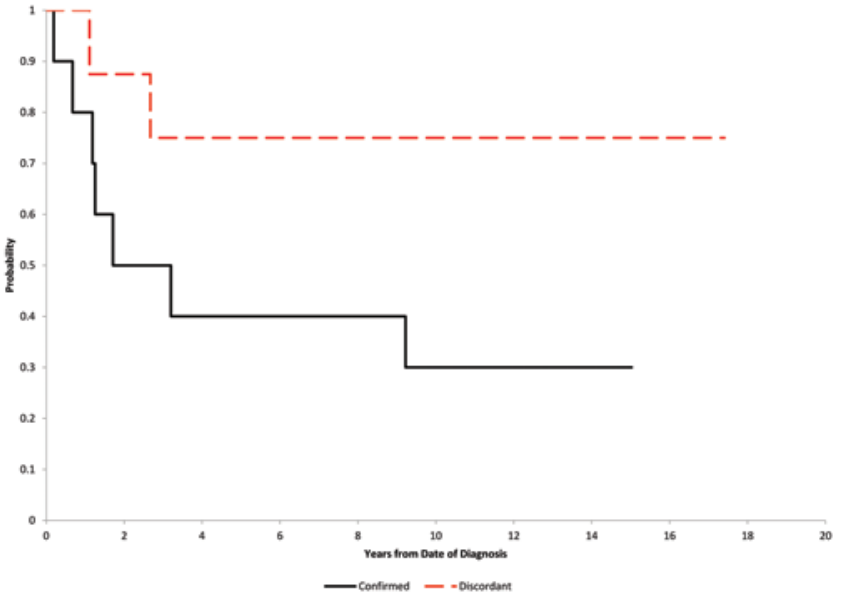

FIG. 3. Distributions of OS between confirmed diagnosis and discordant diagnosis. Figure is available in color online only.

a $32 \% 10$-year overall survival rate for the 24 patients with anaplastic tumors. ${ }^{2}$ There was no long-term survivor in a retrospective cohort from the Armed Forces Institute of Pathology database that identified 36 adult and pediatric cases of primary malignant spinal astrocytic cord tumors diagnosed between 1962 and 2000. ${ }^{21}$ Factors associated with poorer outcome include prodromes shorter than 2 months, Grade IV histology, and dissemination. ${ }^{2}$ The role of surgery in HGG of the spinal cord is still unclear, essentially due to the limited sample size of most series, which precludes a meaningful statistical analysis, and the fact that these lesions are extensive, infiltrative, and usually not amenable to complete resection. To date, CCG-945 is the only prospective study that enrolled patients with malignant astrocytoma of the spinal cord. In the initial report on CCG-945, patients with primary spinal cord HGGs appeared to fare relatively well compared with historical series. ${ }^{1}$ In the initial publication, the 5-year progression-free survival and OS rates were $46 \% \pm 14 \%$ and $54 \pm 14 \%$, respectively, and these results compared favorably with other series from the literature. However, as a consequence of the central pathology review conducted in CCG-945, significant discordance was observed between institutional and central panel consensus review and the EFS and OS for those children with consensus pathology diagnoses of AA or GBM appear less favorable than initially reported. Still, $30 \%$ of patients with confirmed HGG survived, and this survival rate is among the best reported in the literature. The difference in survival for confirmed HGG and discordant diagnoses was not statistically significant, with a 10 -year EFS and OS of 30\% $\pm 12.5 \%$ versus $58.3 \% \pm 18.8 \%(\mathrm{p}=0.108)$ and $30 \% \pm 12.5 \%$ versus $75 \% \pm 14.2 \%(\mathrm{p}=0.0757)$, respectively. However, this is essentially related to the limited sample size and it is likely that a larger sample size would have led to statistically significant results. In the main randomized study, among 172 eligible patients, 24 tumors (14\%) were classified as discordant on single-expert review and 51 (29\%) on consensus review. ${ }^{15}$ Likewise, centralized pathology review in this nonrandomized subgroup of patients with spinal cord tumors led to major changes in diagnoses. Central pathology review determined that a substantial proportion of patients who had enrolled in the study with institutional diagnoses of HGGs in fact bore "discordant" diagnoses, and from the 2 consecutive central reviews conducted, the number of patients with confirmed malignant astrocytoma of the spinal cord fell from 18 to 13 and subsequently to 10. This $44 \%$ discordance rate is much higher than the rate observed in the main randomized study (29\%) and, among the different subgroups analyzed as part of these central reviews, the discordance rate was highest in the patients with primary spinal cord tumors. The reasons for this high discordance rate are only speculative, as no specific explanation was required from or provided by the panel at the time of the central pathology review. Spinal cord biopsies are usually extremely small and may not be representative of the overall lesion. Interpretation of such small specimens may be challenging. In addition, evidence of increased mitotic activity, necrosis, and more particularly endothelial proliferation may be seen in a small proportion of juvenile pilocytic astrocytomas and may lead to inappropriate diagnoses of anaplastic tumor. ${ }^{20}$ Indeed, in the present series, most changes (6 of 12 patients) were seen in patients with an initial diagnosis of AA, whereas no change in histological diagnosis was suggested for patients initially diagnosed with GBM. Whether the consensus pathological diagnosis prevails over institutional diagnosis in spinal cord astrocytomas remains unproven. Case 16 illustrates this issue. In this case, a consensus diagnosis of GBM was achieved, although discrepancies between central experts were obvious (Table 1). Repeat surgery performed 3 months after initial diagnosis led to a total resection of a tumor described as anaplastic ganglioglioma by the experienced institutional neuropathologist. This experience underscores the potential discrepancies between single-institution reports and cooperative series of patients with rare disease. Recent identification of genetic alterations specific to astrocytic tumors may contribute to better segregate high-grade and low-grade tumors. For example, a recent report suggested that combined molecular analysis of BRAF fusion and IDH1 is a sensitive and highly specific approach to separate pilocytic astrocytoma from diffuse astrocytoma. ${ }^{12}$ However IDH1 is not a reliable molecular marker of pediatric $\mathrm{HGG}^{10}{ }^{10}$ and information on specific molecular features underlying HGGs of the spinal cord in the pediatric age group is currently lacking.

Thirty-one institutions participated in CCG-945 and the 10 cases registered over this 5-year period do not reflect the true incidence of malignant astrocytoma of the spinal cord in North America. However, this experience confirms that primary malignant spinal astrocytomas are extremely rare and that reports on such entities should be considered with extreme caution in the absence of central pathology review.

In the CCG-945 study, proliferation index, as assessed by MIB-1 antibody labeling of the nuclear $\mathrm{Ki} 67$ antigen, was found to highly correlate with survival and patients with tumors with labeling indices higher than $36 \%$ had an almost uniformly poor outcome. ${ }^{18}$ Only one patient had had a $\mathrm{Ki} 67$ index higher than $36 \%$ in this series, and he experienced rapid progression of his disease. Although the variance in the proliferation index is large in this cohort, 
the results are in keeping with previous reports on $\mathrm{Ki} 67$ in pediatric HGGs. ${ }^{18}$ The same study also showed that overexpression of p53 in pediatric malignant gliomas was associated with adverse outcome, independently of clinical prognostic factors and tumor histology. ${ }^{17}$ Unlike in adults, studies that looked at deletion of $1 \mathrm{p}$ and $19 \mathrm{q}$ in the CCG945 cohort did not demonstrate a more favorable outcome for patients with tumors that exhibited $1 p$ or $19 q$ deletion versus those that did not. ${ }^{16}$ There has been no dedicated biological study of malignant spinal gliomas and the data presented here are too limited to allow any meaningful conclusions. The relatively high number of $1 p$ or $19 q$ deletions in this cohort (3 of 6 specimens examined) is notable and suggests that these tumors may have a specific biology.

This study was conducted more than 20 years ago and one may argue that these results are of historical interest only, as the criteria used by the reviewers were based on the 1993 revised WHO classification when significant indicators of anaplasia were introduced in the grading of HGG (nuclear atypia, mitotic activity, cellularity, vascular proliferation, and necrosis). There has been in fact little change in the criteria used for grading purposes and data from recent HGG studies in the pediatric population still suggest that a number of patients are erroneously enrolled in these studies. ${ }^{3,4}$

\section{Conclusions}

With only 3 survivors of 10 confirmed cases of HGG, CCG-945 does not provide information on optimal treatment strategies for these malignant spinal cord tumors. Other studies have shown that despite upfront postoperative irradiation, most tumors exhibit local and distant progression within a year. ${ }^{4,14} \mathrm{Up}$ to two-thirds of primary spinal cord malignant gliomas disseminate at recurrence, indicating a need for full CNS prophylaxis. This CCG945 experience has been unique, since subsequent cooperative studies conducted in North America have excluded patients with primary spinal cord tumors. ${ }^{13}$ Given the rarity of these tumors, their diagnostic challenges, and their overall poor prognosis in children, cooperative group studies with centralized review of the histopathological findings are critical for improving outcomes. It is clear that there is also an urgent need to introduce molecular and genetic techniques in the standard investigations used to diagnose these rare tumors.

\section{Acknowledgments}

Biological studies were supported by the CCG grant (U10CA13539), the COG grant (U10CA098543), and the NIH grant R01NS37704 (to Dr. Pollack).

\section{References}

1. Allen JC, Aviner S, Yates AJ, Boyett JM, Cherlow JM, Turski PA, et al: Treatment of high-grade spinal cord astrocytoma of childhood with "8-in-1" chemotherapy and radiotherapy: a pilot study of CCG-945. J Neurosurg 88:215-220, 1998

2. Bouffet E, Pierre-Kahn A, Marchal JC, Jouvet A, Kalifa C, Choux M, et al: Prognostic factors in pediatric spinal cord astrocytoma. Cancer 83:2391-2399, 1998

3. Chastagner P, Kalifa C, Doz F, Bouffet E, Gentet JC,
Ruchoux MM, et al: Outcome of children treated with preradiation chemotherapy for a high-grade glioma: results of a French Society of Pediatric Oncology (SFOP) Pilot Study. Pediatr Blood Cancer 49:803-807, 2007

4. Cohen AR, Wisoff JH, Allen JC, Epstein F: Malignant astrocytomas of the spinal cord. J Neurosurg 70:50-54, 1989

5. DeSousa AL, Kalsbeck JE, Mealey J Jr, Campbell RL, Hockey A: Intraspinal tumors in children. A review of 81 cases. $\mathbf{J}$ Neurosurg 51:437-445, 1979

6. Finlay JL, Boyett JM, Yates AJ, Wisoff JH, Milstein JM, Geyer JR, et al: Randomized phase III trial in childhood high-grade astrocytoma comparing vincristine, lomustine, and prednisone with the eight-drugs-in-1-day regimen. J Clin Oncol 13:112-123, 1995

7. Fouladi M, Hunt DL, Pollack IF, Dueckers G, Burger PC, Becker LE, et al: Outcome of children with centrally reviewed low-grade gliomas treated with chemotherapy with or without radiotherapy on Children's Cancer Group high-grade glioma study CCG-945. Cancer 98:1243-1252, 2003

8. Gilles FH, Tavaré CJ, Becker LE, Burger PC, Yates AJ, Pollack IF, et al: Pathologist interobserver variability of histologic features in childhood brain tumors: results from the CCG-945 study. Pediatr Dev Pathol 11:108-117, 2008

9. Hyder DJ, Sung L, Pollack IF, Gilles FH, Yates AJ, Davis RL, et al: Anaplastic mixed gliomas and anaplastic oligodendroglioma in children: results from the CCG 945 experience. J Neurooncol 83:1-8, 2007

10. Jones C, Perryman L, Hargrave D: Paediatric and adult malignant glioma: close relatives or distant cousins? Nat Rev Clin Oncol 9:400-413, 2012

11. Kleihues P, Burger PC, Scheithauer BW (eds): Histological Typing of Tumours of the Central Nervous System. Berlin: Springer-Verlag, 1993

12. Korshunov A, Meyer J, Capper D, Christians A, Remke M, Witt H, et al: Combined molecular analysis of BRAF and IDH1 distinguishes pilocytic astrocytoma from diffuse astrocytoma. Acta Neuropathol 118:401-405, 2009

13. MacDonald TJ, Arenson EB, Ater J, Sposto R, Bevan HE, Bruner J, et al: Phase II study of high-dose chemotherapy before radiation in children with newly diagnosed high-grade astrocytoma: final analysis of Children's Cancer Group Study 9933. Cancer 104:2862-2871, 2005

14. Merchant TE, Kiehna EN, Thompson SJ, Heideman R, Sanford RA, Kun LE: Pediatric low-grade and ependymal spinal cord tumors. Pediatr Neurosurg 32:30-36, 2000

15. Pollack IF, Boyett JM, Yates AJ, Burger PC, Gilles FH, Davis $\mathrm{RL}$, et al: The influence of central review on outcome associations in childhood malignant gliomas: results from the CCG-945 experience. Neuro Oncol 5:197-207, 2003

16. Pollack IF, Finkelstein SD, Burnham J, Hamilton RL, Yates AJ, Holmes EJ, et al: Association between chromosome 1p and $19 \mathrm{q}$ loss and outcome in pediatric malignant gliomas: results from the CCG-945 cohort. Pediatr Neurosurg 39:114-121, 2003

17. Pollack IF, Finkelstein SD, Woods J, Burnham J, Holmes EJ, Hamilton RL, et al: Expression of p53 and prognosis in children with malignant gliomas. N Engl J Med 346:420-427, 2002

18. Pollack IF, Hamilton RL, Burnham J, Holmes EJ, Finkelstein $\mathrm{SD}$, Sposto R, et al: Impact of proliferation index on outcome in childhood malignant gliomas: results in a multi-institutional cohort. Neurosurgery 50:1238-1245, 2002

19. Przybylski GJ, Albright AL, Martinez AJ: Spinal cord astrocytomas: long-term results comparing treatments in children. Childs Nerv Syst 13:375-382, 1997

20. Rutka JT, Kuo JS, Carter M, Ray A, Ueda S, Mainprize TG: Advances in the treatment of pediatric brain tumors. Expert Rev Neurother 4:879-893, 2004

21. Santi M, Mena H, Wong K, Koeller K, Olsen C, Rushing EJ: 
Spinal cord malignant astrocytomas. Clinicopathologic features in 36 cases. Cancer 98:554-561, 2003

22. Sposto R, Ertel IJ, Jenkin RD, Boesel CP, Venes JL, Ortega JA, et al: The effectiveness of chemotherapy for treatment of high grade astrocytoma in children: results of a randomized trial. A report from the Childrens Cancer Study Group. J Neurooncol 7:165-177, 1989

\section{Disclosures}

The authors report no conflict of interest concerning the materials or methods used in this study or the findings specified in this paper.

\section{Author Contributions}

Conception and design: Bouffet, Allen, Yates, Gilles, Burger,
Davis, Becker, Pollack, Finlay. Acquisition of data: Bouffet, Yates, Gilles, Burger, Davis, Becker, Pollack, Finlay. Analysis and interpretation of data: Bouffet, Allen, Boyett, Burger, Davis, Pollack, Finlay. Drafting the article: Bouffet. Critically revising the article: Bouffet, Allen, Boyett, Pollack, Finlay. Statistical analysis: Boyett. Study supervision: Finlay.

\section{Supplemental Information}

Previous Presentations

This work was presented in part at the 11th International Symposium on Pediatric Neuro-Oncology, Boston, Massachusetts, June 13-16, 2004.

\section{Correspondence}

Eric Bouffet, Hospital for Sick Children, 555 University Ave., Toronto, ON M5G 1X8, Canada. email: eric.bouffet@sickkids.ca. 